\title{
Building cognizance rule knowledge for fault diagnosis based on fuzzy rough sets ${ }^{1}$
}

\author{
$\mathrm{Xi}-\mathrm{Xu} \mathrm{He} \mathrm{e}^{\mathrm{a}, \mathrm{c}, \mathrm{d}, *}$, Lei-ting Chen ${ }^{\mathrm{a}, \mathrm{b}, \mathrm{d}}$ and Hai-tao Jia ${ }^{\mathrm{a}}$ \\ ${ }^{a}$ School of Computer Science and Engineering, University of Electronic Science and Technology of China, \\ Sichuan, China \\ ${ }^{\mathrm{b}}$ Dongguan Institute of Information Engineering, University of Electronic Science and Technology of China, \\ Sichuan, China \\ ${ }^{\mathrm{c}}$ Center of Information, University of Electronic Science and Technology of China Chengdu, Sichuan, China \\ ${ }^{\mathrm{d}}$ Provincial Key Laboratory of Digital Media Chengdu, Sichuan, China
}

\begin{abstract}
With the continuous development of huge systems, dependence on the system is continually increasing. The failure of such systems will cause huge losses. The reason for system failure is often unclear, so that inconsistency and uncertainty between fault data will appear. In the actual application process, there is a process of change. If it is possible to predict the failure probability from the monitoring parameters, it will be very beneficial to system troubleshooting. Therefore, this paper proposes a new recognition algorithm based on fuzzy rough sets, in order to adapt to the processing of uncertain fault detection data. Additionally, the optimal direction of the dynamic information entropy increment is used to predict the fault information. This can quickly find the faults and provide important information for fault detection. It is verified that the proposed algorithm can improve the early warning and the accuracy of fault diagnosis information systems in the fault simulation analysis of a diesel engine.
\end{abstract}

Keywords: Rough set, fuzzy rough set, attribute importance, fault diagnosis, cognizance rule

\section{Introduction}

With the quick development of modern industry and scientific technology, and with the large-scale, high-speed and automatic trends in modern equipment, people have become more concerned about the safety and reliability of equipment. In recent years, data techniques have become increasingly important to the development of modern industrial technology [19]. Faults of modern industrial production will result in disasters, once the fault has occurred. Recently, the

\footnotetext{
${ }^{1}$ This paper was supported by EPR Major Program of Guangdong Province of China, and Research and application of key technology of residents' health services based on Cloud Computing (No. 2012A090300001).

${ }^{*}$ Corresponding author. Xi-Xu He. Tel.: +86 18080987271; Fax: +28 83204056 210; E-mail: HL@uestc.edu.cn.
}

disastrous accidents caused by critical equipment faults have inspired much research on equipment diagnosis, that have resulted in an emerging field of fault diagnosis of equipment, engineering structure and technological processes [26]. S. Yin introduces the application of data-driven methods in industrial monitoring, which has occurred in the last twenty years and promotes the rapid development of industrial automation [18]. Fault detection and diagnosis technology has been proposed as a new method to improve the reliability and safety of systems so that fault detection and diagnosis technologies within dynamic systems have become a rapid field of development. Existing fault diagnosis methods can be roughly divided into model-based methods, knowledge-based methods and methods based on image processing. 
The residual between the measurable information and prior system information expressed by a model is used in the model-based method, which can achieve fault diagnosis by analyzing and processing the residual. This method must establish the mathematical model of diagnosed objects, and estimate the parameters using detective signals or by reconstructing the system state. The test for state estimation residual sequences and identification are used to forecast, locate and quantify the fault [24].

Due to the connection between the amplitude, phase, frequency and correlation of system I/O signals and the fault source, the fault can be detected and isolated by the application of a signal processing method and a characteristic extraction method after the fault has occurred. This method based on signal analysis theory provides multiple eigenvectors in the time domain and frequency domain, and exploits the connection between eigenvectors and fault sources to analyze and process the system signal so that the position of fault sources can be determined [9].

The knowledge-based method exploits expert diagnostic knowledge and diagnostic objects without establishing a quantitative mathematical model applied the fuzzy logic method to the adaptive adjustment of residual thresholds [7, 16]. M. Hemza proposed the automatic detection of faults using a neural network [11]. A. Bellini adopted a method based on pattern recognition to complete the automatic detection of non-linear system faults [1]. S. Yin derives the framework of data-driven design, which can be used for the monitoring of large-scale industrial processes [22]. S. Yin introduces an improved PLS (IPLS) method, which provides a high detection rate and can effectively diagnose the faults of KPI [20]. The expert system is also one algorithm of fault detection systems [13].

In spite of the fantastic results from state-of-the-art research on fault diagnosis, unsolved problems remain, as follows:

The unknown mechanism of faults from diagnostic equipment may result in ambiguity in the external manifestation of faults and unclear system states expressed by diagnostic information. Different faults may demonstrate similar performance, whereas the same fault may express differently at different times or under different environmental conditions. The ambiguity and inconsistency among failure data will have a major impact on the operating results of fault detection systems, which is not advantageous to the application of fault detective technology.
Shorter time of fault diagnosis will result in smaller losses caused by accidents. Therefore, the development of rapid diagnostic technology is very important, particularly rapid diagnostic technology that does not reduce the accuracy of fault diagnosis.

In this paper, a method based on cognitive rule extraction is used to achieve dynamic analysis and intelligent cognition of informative systems, monitored by a realtime system so that the fault is found more quickly. The continuous change of diagnostic information is considered, and the rule extraction is achieved by a cognitive rule in which the fuzzy rough set is adopted. The simulative analysis of a diesel engine fault demonstrates that the intelligent cognitive rules can achieve earlier warning regarding fault diagnosis information systems, and the improved accuracy of such systems.

This paper is organized as follows. Section 2 presents fuzzy rough set theory. Section 3 details the cognizance rule generation. Section 4 presents the simulation of a diesel engine fault for evaluation of the proposed method.

\section{Fuzzy rough set theory}

As an extended rough set, fuzzy rough sets introduce fuzzy set theory, so that the rough set can effectively handle complex uncertain problems. The fuzzy decision table is defined in this section.

Definition 1. Let the fuzzy decision table be defined as $D T=(U, A=C \cup D, V, f) . U$ is the discourse domain; $A$ is the fuzzy attribute set, which includes condition attribute set $C=\left\{A^{1}\right.$, $\left.A^{2}, \cdots, A^{m}\right\}$ and decision attribute set $D=\left\{A^{m+1}\right\}$, in which $A^{j}$ is the fuzzy attribute on domain $U$. $U$ can be divided into $p_{j}$ fuzzy equivalence classes, namely $F\left(A^{j}\right)=\left\{F_{1}^{j}, F_{2}^{j}, \cdots, F_{p_{j} j}\right\} . F_{i}^{j}(1 \leq$ $\left.i \leq p_{j}, j=1,2, \cdots, m+1\right)$ is a fuzzy set in the domain $U$.

Since fuzzy rough sets introduce the fuzzy sets, the equivalent class, as well as the upper and lower approximation concepts in classical rough set theory are also extended to the fuzzy equivalence class, vague upper approximation, and lower fuzzy approximation, respectively.

Definition 2. Let $R$ be defined as a fuzzy equivalence relation on domain $U$, namely the fuzzy attribute. For $x \in U$ :

$$
\mu_{[\mathrm{x}]_{R}}=\mu_{R}(x, y)
$$


Represents the fuzzy equivalence class of object $x$. This represents the aggregation of all adjacent elements of $x$ in domain $U$, and is a fuzzy set.

Definition 3. For the fuzzy decision table $D T=$ $(U, A=C \cup D, V, f), R$ is a fuzzy attribute on domain $U$, and $\mathrm{X} \subseteq \mathrm{U}$. The lower approximation $\underline{R} X$ membership and upper approximation $\bar{R} X$ membership about $\mathrm{R}$, respectively, are as follows:

$$
\begin{array}{r}
\mu_{\underline{R} X}\left(F_{i}\right)=\inf _{x \in U} \max \left\{1-\mu_{F i}(x), \mu_{X}(x)\right\} \forall i \\
\mu_{\bar{R} X}\left(F_{i}\right)=\sup _{x \in U} \max \left\{\mu_{\mathrm{F}_{\mathrm{i}}}(x), \mu_{X}(x)\right\} \forall i
\end{array}
$$

where $\underline{R} X$ and $\bar{R} X$ are both fuzzy set on $U$. In Formula $3, \mathrm{~F}_{i}$ is a fuzzy equivalence class on $U$ about $R$ division, that $\mathrm{F}_{i} \subseteq \mathrm{U} / \mathrm{R}$. The fuzzy lower approximation and upper approximation can be defined as follows:

$$
\begin{aligned}
& \mu_{\underline{R} X}(x)=\sup _{F_{i} \in U / \mathbb{R}} \min \left(\mu_{F_{\mathrm{i}}}(x), \inf _{y \in U} \max \left\{1-\mu_{\mathrm{F}_{\mathrm{i}}}(\mathrm{y}), \mu_{X}(y)\right\}\right) \\
& \mu_{\bar{R} X}(x)=\sup _{F_{i} \in U / R} \min \left(\mu_{F_{i}}(x), \inf _{y \in U} \min \left\{\mu_{\mathrm{F}_{i}}(\mathrm{y}), \mu_{X}(y)\right\}\right)
\end{aligned}
$$

The fuzzy lower approximation and upper approximation constructed binary pair $\langle\underline{R} X, \bar{R} X\rangle$ is designated the fuzzy rough set.

Definition 4. In rough set theory, the attribute set A in the domain partition can be expressed as follows:

$$
\mathrm{U} / \mathrm{A}=\otimes\{U / \alpha \mid \alpha \in \mathrm{A}\}
$$

where $\otimes$ denotes $S_{1} \otimes S_{2}=\left\{X \cap Y \mid X \in S_{1}, Y \in\right.$ $\left.\mathrm{S}_{2}, \mathrm{X} \cap \mathrm{Y} \neq \emptyset\right\}$

$$
\begin{aligned}
\text { If } \mathrm{A}= & \left\{\alpha_{1}, \alpha_{2}, \cdots \alpha_{m}\right\}, \\
U / A= & \left\{X_{1_{i 1}} \cap \mathrm{X}_{2_{i 2}} \cap \cdots \cap \mathrm{X}_{1_{i m}} \mid \mathrm{X}_{1_{i 1}} \in U / \alpha_{1}, \mathrm{X}_{1_{i 2}}\right. \\
& \left.\in U / \alpha_{2}, \cdots, \mathrm{X}_{1_{\text {im }}} \in U / \alpha_{m}\right\}
\end{aligned}
$$

Through the definition of the fuzzy equivalence class of attribution set $A$, above, the membership function of an object belonging to such a fuzzy equivalence classes can be defined as follows:

$$
\begin{aligned}
& \mu_{F_{1} \cap F_{2} \cap \cdots \cap F_{m}}(x)=\mu_{F_{1}}(x) \wedge \mu_{F_{2}}(x) \wedge \cdots \wedge \mu_{F_{m}}(x) \\
& =\min \left(\mu_{F_{1}}(x), \mu_{F_{2}}(x), \cdots, \mu_{F_{m}}(x)\right)
\end{aligned}
$$

where $F_{1} \cap F_{2} \cap \cdots \cap F_{m}$ is one fuzzy equivalence class of $U / A$.

In classical rough set theory, the positive region is defined as the union of the lower approximation.
According to this rule, the membership function that one object belongs to in the fuzzy positive region of a rough fuzzy rough set can be defined.

Definition 5. Given a fuzzy decision table $D T=$ $(U, \underset{\sim}{A}=C \cup D, V, f), P \in C, Q \in D$, define

$$
\mu_{\operatorname{pos}_{P}(Q)}(x)=\sup _{X \in U / Q} \mu_{\underline{P}(X)}(x)
$$

to obtain the membership degree that $x$ belongs to in the fuzzy positive domain. Based on the definition of the fuzzy positive domain, a new dependent function in the fuzzy rough set can be defined as follows:

$$
\gamma_{\mathrm{P}}^{\prime}(\mathrm{Q})=\left|\mu_{\operatorname{pos}_{P}(\mathrm{Q})}(x)\right| /|\mathrm{U}|=\sum_{x \in \mathrm{U}} \mu_{\operatorname{pos}_{P}(\mathrm{Q})}(x) /|\mathrm{U}|
$$

This indicates the degree to which attribute $Q$ depends on attribute $P$.

The basic theory of fuzzy rough set is detailed above. Based on this theory, an algorithm is proposed to obtain the computational cognitive rules so as to improve recognition rate and speed.

\section{Cognizance rule development}

The unknown mechanism of faults from diagnostic equipment may result in ambiguity in the external manifestation of faults and unclear system states expressed by diagnostic information. Different faults may demonstrate similar performance, whereas the same fault may express differently at different times or under different environmental conditions. The ambiguity and inconsistency in failure data will have great impact on the operating results of the fault detection system, which is not advantageous for the application of fault detective technology. Shorter detection times may reduce losses from accidents, and has thus become a primary research focus in the field. The cognitive rule intelligent extraction algorithm based on a rough set can deal with uncertain fault detection data, and detect fault information based on the optimum direction of the dynamic entropy increment to improve the accuracy and efficiency of detection.

For complex systems, many states can be expressed. There is inconsistency in these statistics under different environmental conditions. For example, for some states, a characteristic may be consistent under environmental conditions, but in some contradictory situations, system faults may occur after environmental changes. Therefore, the inevitable connection among system faults, 


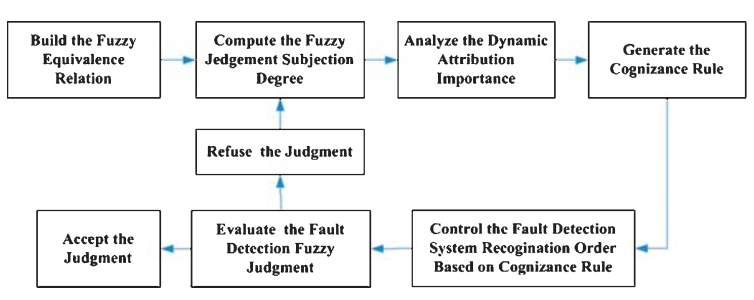

Fig. 1. The framework for cognizance rule generation.

states and different environments should be discussed dynamically to better obtain connection information between system states and faults. Based on the direction of maximum entropy, the cognitive rule states that the rule can be obtained in conjunction with the maximum dynamic information entropy of the fault detection system state, which can be analyzed under different environmental conditions.

The framework of cognizance rule acquirement is shown in Fig. 1.

The steps to achieve cognizance rule generation are as follows:

Build the fuzzy equivalence relation, which can be used to evaluate the correlation degree of two information system samples in the universe.

Determine the fuzzy judgment subjection degree, which must suit the information judgment table data. The cognizance rule defines the formula as follows:
The card is the member number of the set; a threshold can be set by the user, such as 0.8 .

After computing all no-value attributes of dynamic importance, the cognizance rule can be generated based on the dynamic attribution importance. The cognizance rule form is as follows:

$$
\left(a_{i}, v_{i}\right) \cap\left(a_{i+1}, v_{i+1}\right) \cap \cdots \cap\left(a_{i+n}, v_{i+n}\right) \rightarrow a_{j}
$$

Then, the system can arrange the system state information in detection order for fault diagnosis.

After obtaining the new system states, the fault diagnosis system evaluates the fuzzy judgment. If the judgment subjection degree is beyond the threshold, the system accepts the judgment. Otherwise, the system regenerates the cognizance rule for the next information index in order.

\section{Simulation}

To evaluate the cognizance rule generation framework for fault detection, this paper presents a simulation which compares the cognizance rule to attribution importance. Attribution importance is the statistical measurement, and is described as follows:

$$
\operatorname{Sig}=\frac{\operatorname{card}\left(P O S_{U /\left\{\left\{a_{j}\right\}\left\{a_{i} \cdots a_{i+n}\right\}\right\}}(\widehat{D})\right)-\operatorname{card}\left(\operatorname{POS}_{U /\left\{\left\{a_{i} \cdots a_{i+n}\right\}\right.}(\widehat{D})\right)}{\operatorname{card}(\operatorname{POS}(\widehat{D}))}
$$

$$
\widehat{D}=\min \left(\exp \left(\frac{x_{i}-\bar{x}_{i}}{4 \delta^{2}}\right)\right), i=1,2, \ldots, n
$$

where $x_{i}$ is the value $i$ of the attribute, and $\bar{x}_{i}$ is the mean value of the attribute. The variable $\delta$ is the variance or all attribute values.

Analyze the dynamic attribution importance, which considers the already-described attribute information.

$$
\operatorname{FSig}_{d}\left(a_{j} \mid R\left(x_{p}\right)\right)=\frac{\operatorname{card}\left(F P O S_{U /\left\{\left\{a_{j}\right\}\left\{a_{i}, \ldots a_{j-1}\right\}\right\}}(\widehat{D})\right)}{\operatorname{card}\left(F P O S_{U /\left\{a_{i}, \ldots a_{j-1}\right\}}(\widehat{D})\right)}
$$

where the FPOS is the fuzzy equivalence relation to the position, which can be expressed as:

$$
F_{P O S} S_{U /\left\{a_{1}, \cdots, a_{j-1}\right\}}=\mu_{p o s_{\left\{a_{1}, \cdots, a_{j-1}\right\}}(\mathrm{U})}(x)>\text { threshold }
$$

This simulation employs diesel engine malfunction as the fault detection system, which can discover eight malfunctions using eight detection parameters. These eight malfunctions are as follows: $100 \%$ offering $\left(T_{1}\right)$, $75 \%$ offering $\left(T_{2}\right), 25 \%$ offering $\left(T_{3}\right)$, idle consumption $\left(T_{4}\right)$, pin valve block $\left(T_{5}\right)$, valve jam $\left(T_{6}\right)$, pin valve leak $\left(T_{7}\right)$ and excess oil valve invalidation $\left(T_{8}\right)$. The eight detection parameters are as follows: greatest pressure $\left(P_{1}\right)$, hypo-greatest pressure $\left(P_{2}\right)$, wave range $\left(P_{3}\right)$, ascending edge width $\left(P_{4}\right)$, wave width $\left(P_{5}\right)$, greatest aftereffect width $\left(P_{6}\right)$, wave area $\left(P_{7}\right)$ and spout pressure $\left(P_{8}\right)$. According to the maximum and minimum detected by each sensor and normalized to the $[-1,1]$ space, a data set will be recorded, which is then adapted to the output of various classifiers (or sensors). The diesel engine malfunction judgment information is listed in Table 1. 
Table 1

Diesel engine malfunction judgment information table

\begin{tabular}{|c|c|c|c|c|c|c|c|c|}
\hline & $P_{1}$ & $P_{2}$ & $P_{3}$ & $P_{4}$ & $P_{5}$ & $P_{6}$ & $P_{7}$ & $P_{8}$ \\
\hline$T_{1}$ & 0.9325 & 1.000 & 1.000 & -0.4526 & 0.3895 & 1.000 & 1.000 & 1.000 \\
\hline$T_{2}$ & -0.4571 & -0.2854 & -0.9024 & -0.9121 & -0.0841 & 1.000 & -0.2871 & 0.5647 \\
\hline$T_{3}$ & 0.5134 & 0.9413 & 0.9711 & -0.4187 & 0855 & 0.8546 & 0.9478 & 0.9512 \\
\hline$T_{4}$ & 0.1545 & 0.1564 & -0.500 & -0.6571 & -0.3333 & -0.6667 & -0.3333 & -0.500 \\
\hline$T_{5}$ & 0.1764 & 0.7648 & 0.4259 & -0.6472 & -0.0563 & 0.1726 & 0.5151 & 0.4212 \\
\hline$T_{6}$ & -0.6744 & -0.4541 & -0.8454 & 1.000 & -0.8614 & -0.6714 & -0.6279 & -0.6785 \\
\hline$T_{7}$ & 0.4647 & 0.5710 & 0.0712 & -0.7845 & -0.2871 & 0.8915 & 0.6553 & 0.6152 \\
\hline$T_{8}$ & 0.6818 & 1.000 & -0.625 & -0.8426 & -0.6215 & -0.1574 & 1.000 & 0.7782 \\
\hline
\end{tabular}

By analyzing this table, the statistic character can be determined. There are three cluster parameter centers:

$$
\begin{aligned}
& {\left[V_{\text {mean } 1}=0.79, V_{\mathrm{var} 1}=0.22\right],} \\
& {\left[V_{\text {mean } 2}=-0.06, V_{\mathrm{var} 2}=0.21\right]} \\
& {\left[V_{\text {mean } 3}=0.66, V_{\mathrm{var} 3}=0.15\right] .}
\end{aligned}
$$

The rule following fault detection information can be obtained from the intelligent extraction step and cognitive rule equations in Section 3. For example, in the known state $\mathrm{P} 1$ conditions and according to the attribute importance, the detection rule is as follows.

$$
P_{1} \rightarrow P_{6} \rightarrow P_{3} \rightarrow P_{8} \rightarrow P_{2} \rightarrow P_{7} \rightarrow P_{4} \rightarrow P_{5}
$$

The cognitive rules based on the fuzzy rough set maximum dynamic information entropy in this paper are expressed as follows:

$$
\begin{aligned}
& P_{1}=V_{\text {mean } 1} \rightarrow P_{3} \rightarrow P_{5} \rightarrow P_{4} \rightarrow P_{6} \rightarrow P_{8} \rightarrow P_{2} \rightarrow \begin{array}{r}
P_{7} \\
(16)
\end{array} \\
& P_{1}=V_{\text {mean } 2} \rightarrow P_{3} \rightarrow P_{5} \rightarrow P_{4} \rightarrow P_{6} \rightarrow P_{8} \rightarrow P_{2} \rightarrow \begin{array}{r}
(17) \\
(17)
\end{array} \\
& P_{1}=V_{\text {mean } 3} \rightarrow P_{4} \rightarrow P_{3} \rightarrow P_{5} \rightarrow P_{6} \rightarrow P_{8} \rightarrow P_{2} \rightarrow P_{7}
\end{aligned}
$$

These cognitive rules distinguish the analysis direction of maximum dynamic information entropy of the fault detection system within the different initial values in state P1. Such directions are more suitable for the cognitive mechanism of the biosphere, so the fault detection accuracy and efficiency can be determined.

Results indicate that cognizance rules can offer more detail in order to control fault detection processing more intelligently and efficiently. The simulation system tests different dates with variant system noise to evaluate the result of the cognizance rules and attribute importance. Figure 2 shows the system judgment results with 0.21 system noise.

Figure 2 shows the system judgment results with 0.1 system noise.

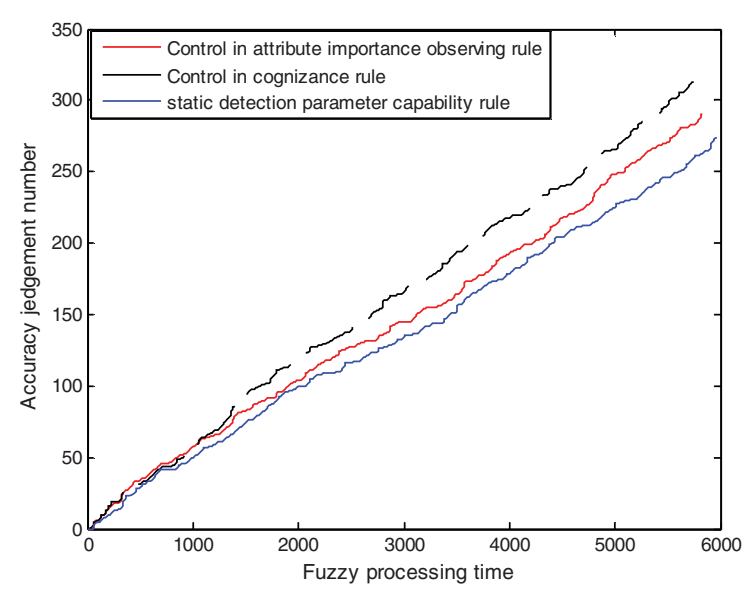

Fig. 2. Discrimination results for 1,000 malfunction samples with 0.21 system noise.

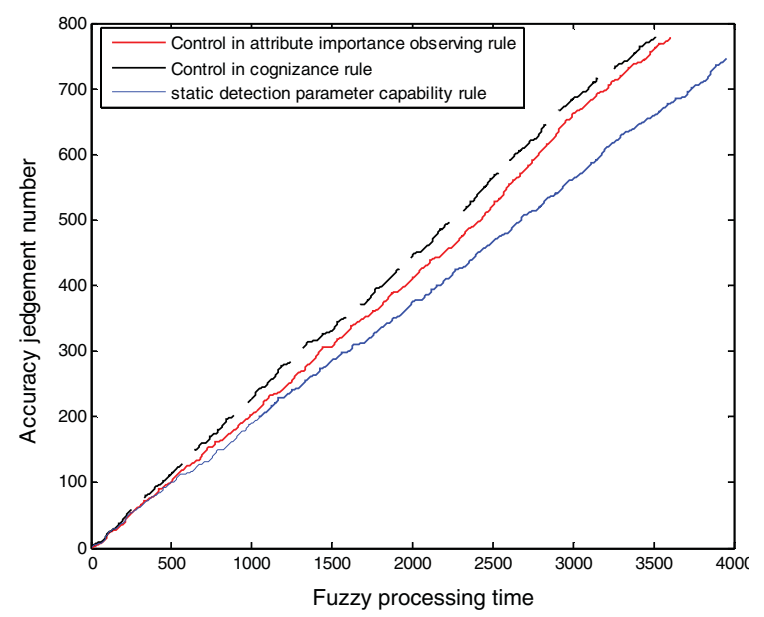

Fig. 3. Discrimination results for 1,000 malfunction samples with 0.1 system noise.

Figure 3 shows the system judgment results on system noise 0.1 .

This paper uses the Monte Carlo simulation method to test 300 experiments in order to evaluate the accuracy 


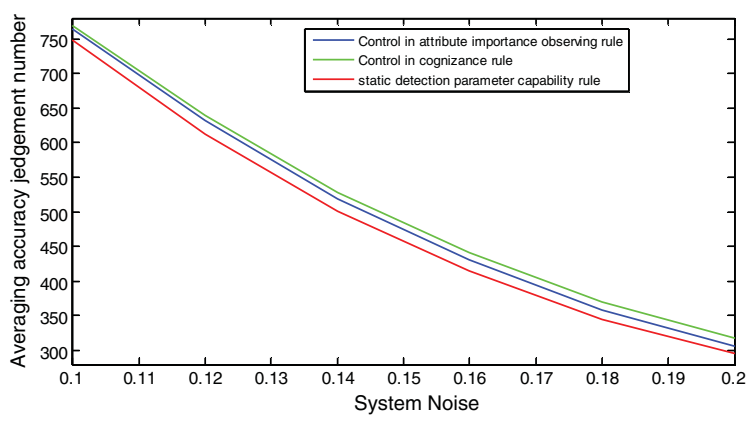

Fig. 4. Discrimination accuracy result for 1,000 malfunction samples with $[0.1,0.2]$ system noise, averaging 300 Monte Carlo simulation.

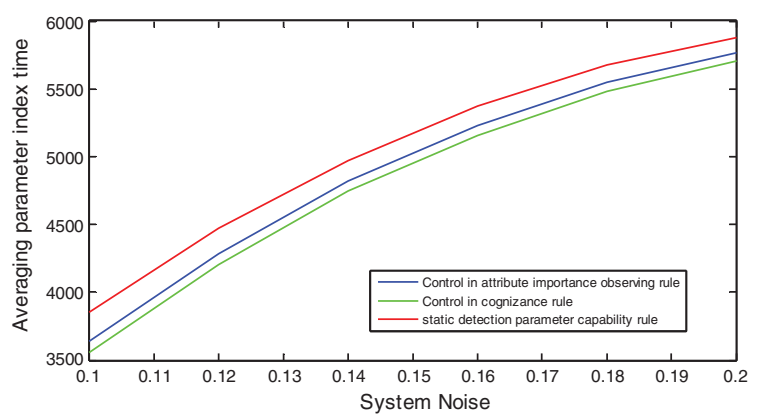

Fig. 5. The discrimination efficiency result for 1000 malfunction sample on $[0.1,0.2]$ system noise averaging 300 Monte Carlo simulation.

and efficiency of the variant index rule. Figure 4 shows discrimination accuracy result for 1,000 malfunction samples with $[0.1,0.2]$ system noise, averaging 300 Monte Carlo simulation. Figure 5 shows the discrimination efficiency result for 1000 mal-function sample on $[0.1,0.2]$ system noise averaging 300 Monte Carlo simulation.

For diesel engine fault detection data, different signal-to-noise of Monte Carlo simulation experiments show that the cognitive rules based on fuzzy rough set maximum dynamic information entropy discovery system failure better. Within the same signal-to-noise ratio data, the accuracy of the fault detection analysis based on maximum dynamic information entropy from cognitive rule that is higher than from regular attribute rules. This method could improve the fault detection efficiency. The cognitive rules based on fuzzy rough set maximum dynamic information entropy are also adaptive to deal with uncertain data for fault detection in more complicated systems.

\section{Conclusions}

Due to the unknown mechanisms of faults in complex systems and the ambiguity and the inconsistency of system states and fault diagnosis, this paper proposes a method based on cognitive rule extraction to achieve dynamic analysis and intelligent cognition of informative systems monitored by a real-time system, so that the fault is found more quickly. Based on the direction of maximum entropy, the cognitive rule can improve the recognition rate resulting in earlier fault detection. The continuous change of diagnostic information is considered, so the rule extraction is achieved by cognitive rules which adopt the fuzzy rough set. The simulative analysis of a diesel engine fault indicates that the intelligent cognitive rules can achieve earlier detection in fault diagnosis information systems, with improved accuracy.

\section{References}

[1] A. Bellini, F. Filippetti, C. Tassoni and G.-A. Capolino, Advances in diagnostic techniques for induction machines, IEEE Trans Ind Electron 55 (2008), 4109-4126.

[2] A. Zeng, D. Pan, Q.L. Zheng and H. Peng, Knowledge acquisition based on rough set theory and principal component analysis, IEEE Intelligent Systems 21(2) (2006), 78-85.

[3] C. Cornelis, M.D. Cock and A.M. Radzikowska, Vaguely quantified rough sets, Proc Rough Sets, Fuzzy Sets, Data Mining Granular Compute (Lecture Notes in Artificial Intelligence) 4482 (2007), 87-94.

[4] C.Y. Wang, Type-2 fuzzy rough sets based on extended t-norms, Information Sciences 305 (2015), 165-183.

[5] D.S. Yeung, D.G. Chen, E.C.C. Tsang, J.W.T. Lee and X.Z. Wang, On the generalization of fuzzy rough sets, IEEE Trans Fuzzy Syst 13 (2005), 343-361.

[6] D. Lynn, V. Nele, C. Chris and G. Llus, Comprehensive study of implicator-conjunctor-based and noise-tolerant fuzzy rough sets: Definitions, properties and robustness analysis, Fuzzy Sets and Systems (2014) 275 (2015), 1-38.

[7] E. Bouchikhi and E. Houssin, Induction machine faults detection using stator current parametric spectral estimation, Mechanical Systems and Signal Processing 52-53 (2015), 447-464.

[8] J.S. Mi and W.X. Zhang, An axiomatic characterization of a fuzzy generalization of rough sets, Inf Sci 160 (2004), 235-249.

[9] K.S. Lee and T.G. Park, Robust fault detection observer design under fault sensitivity constraints, Journal of the Franklin Institute 352 (2015), 1791-1810.

[10] L. Maryam, S. Shiratuddin, M.F. Wong and K. Wai, Utilising fuzzy rough set based on mutual information decreasing method for feature reduction in an image retrieval system, Lecture Notes in Electrical Engineering 313 (2015), 177-184.

[11] M. Hemza, B. Omar and B. Djamel, Sliding mode based fault detection, reconstruction and fault tolerant control scheme for motor systems, ISA Transactions 57 (2015), 340-351.

[12] M. Quafafou, $\alpha$-RST: A generalization of rough set theory, Inf Sci 124 (2000), 301-316. 
[13] N. Talebi, M.A. Sadrnia and A. Darabi, Fault detection of wind energy conversion systems using recurrent neural networks, International Journal of Sustainable Energy 34 (2015), 52-70.

[14] N.M. Parthalain, Q. Shen and R. Jensen, A distance measure approach to exploring the rough set boundary region for attribute reduction, IEEE Transactions on Knowledge and Data Engineering 22 (2010), 305-317.

[15] Q. Shen and R. Jensen, Selecting informative features with fuzzy-rough sets and its application for complex systems monitoring, Pattern Recognit 37 (2004), 1351-1363.

[16] R. Rubini and U. Meneghetti, Application of the envelope and wavelet transform analysis for the diagnosis of incipient faults in ball bearings, Mech Syst Signal Process 15 (2001), 287-302.

[17] R. Jensen and Q. Shen, Fuzzy-rough sets assisted attribute selection, IEEE Transactions on Fuzzy Systems 15 (2007), 73-89.

[18] S. Yin, X.W. Li, H.J. Gao and K. Okyay, Data-based techniques focused on modern industry: An overview, IEEE Transactions on Industrial Electronics 62 (2015), 657-667.

[19] S. Yin and Z. Huang, Performance monitoring for vehicle suspension system via fuzzy positivistic C-means clustering based on accelerometer measurements, IEEE/ASME Transactions on Mechatronics 20(5) (2015), 2613-2620.

[20] S. Yin, X.P. Zhu and O. Kaynak, Improved PLS focused on key-performance-indicator-related fault diagnosis, IEEE Transactions on Industrial Electronics 62 (2015), 1651-1658.

[21] S.Y. Zhao, E.C.C. Tsang and D.G. Chen, The model of fuzzy Variable precision rough sets, IEEE Transactions on Fuzzy Systems 17 (2009), 451-467.
[22] S. Yin, S.X. Ding, X.C. Xie and H. Luo, A review on basic datadriven approaches for industrial process monitoring, IEEE Transactions on Industrial Electronics 61 (2014), 6418-6428.

[23] S.Y. Zhao, E.C.C. Tsang, D.G. Chen and X.Z. Wang, Building a rule-based classifier-A fuzzy-rough set approach, IEEE Transactions on Knowledge and Data Engineering 22 (2010), 624-638.

[24] W. Kochaski, M. Boeff, Z. Hashemiyan, W.J. Staszewski and P.K. Verma, Modelling and numerical simulations of in-air reverberation images for fault detection in medical ultrasonic transducers: A feasibility study, Journal of Sensors 2015 (2015), 14.

[25] Y. Li, S.C.K. Shiu and S.K. Pal, Combining feature reduction and case selection in building CBR classifiers, IEEE Transactions on Knowledge and Data Engineering 18 (2006), 415-429.

[26] Z.L. Li, R. Outbib, S. Giurgea, D. Hissel and Y.D. Li, Fault detection and isolation for polymer electrolyte membrane fuel cell systems by analyzing cell voltage generated space, Applied Energy 148 (2015), 260-272.

[27] Z.W. Li and T.S. Xie, Roughness of fuzzy soft sets and related results, International Journal of Computational Intelligence Systems 8 (2015), 278-296.

[28] Z. Pawlak, Rough sets, Int J Comput Inf Sci 11 (1982), 341-356.

[29] Z. Pawlak and A. Skowron, Rough sets: Some extensions, Information Sciences 177 (2007), 28-40. 